\author{
Onno A. van Nierop \\ Aadjan van der Helm \\ Kees J. Overbeeke \\ Tom J.P. Djajadiningrat
}

\title{
A natural human hand model
}

Published online: 3 October 2007

(C) Springer-Verlag 2007

O.A. van Nierop ( $)$ A. van der Helm Delft University of Technology, Faculty of Industrial Design Engineering, ID-StudioLab,

Landbergstraat 15, 2628 CE Delft, The Netherlands

o.a.vannierop@io.tudelft.nl

K.J. Overbeeke

Eindhoven University of Technology,

Faculty of Industrial Design,

Den Dolech 2, 5612 AZ Eindhoven

T.J.P. Djajadiningrat

Philips N.V. Eindhoven,

Design Center

\author{
Abstract We present a skeletal \\ linked model of the human hand \\ that has natural motion. We show \\ how this can be achieved by intro- \\ ducing a new biology-based joint \\ axis that simulates natural joint \\ motion and a set of constraints that \\ reduce an estimated 150 possible \\ motions to twelve. The model is \\ based on observation and litera- \\ ture. \\ To facilitate testing and evaluation, \\ we present a simple low polygon \\ count skin that can stretch and \\ bulge. To evaluate we first introduce \\ a hand-motion taxonomy in a two- \\ dimensional parameter space based \\ on tasks that are evolutionary linked
}

to the environment. Second, we discuss and test the model.

The appendix shows motion sequences of the model and the real hand. Animations can be fetched from our website.

Keywords Natural hand model . Evolution · Taxonomy · Anatomy . Biomechanics $\cdot$ Joint model

\section{Introduction}

The human hand is an inseparable part of the self and thereby a manifestation of the self in the world. Its motions have natural boundaries that are fine tuned to the evolutionary developed abilities of man to grasp, use tools, to gesture and to caress.

The human hand shows full natural motion in its dexterous functionality; drawing, model making, playing musical instruments, sustaining speech etc., all sensitive abilities for subtle manipulation.

As designers and hand modelers we want to stress that modeling the human hand has to be natural as that benefits all its applications, so making sense are all those models that have a biological basis, also argued by Tsang et al. [32].

This biological basis has to be subtle and expressive, which means detailed in its technicalities. "Short cuts" that "look good" [4,27], but ignore biological subtleties contribute little to natural hand modeling. The hand modelers community cannot but add to and build on work previously done, but relevant old work is sometimes simply ignored and other work uncritically accepted and used.

Various authors emphasize different aspects e.g., Fleming [10] concentrates on the highly realistic appearance of a static hand posture (Fig. 1a [3]) in which no motion is involved, but the appearance can be an example to skin modelers. Rijpkema \& Girard [28] focus on grasp and pinch while leaning on very superficial, anatomical assumptions (Fig. 1b) but contribute to computer animation. Rohling et al. [29] devise a model to fit their robot-hand with "fingers" and a "thumb" (Fig. 1c), in which we found no other references to biology.

For our work we are indebted to Hummels [15] for her initiating ideas based on gesture research. The involvement of Magnenat-Thalman gave direction [23, 25] 


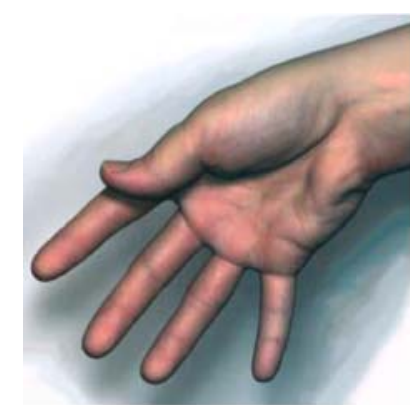

a

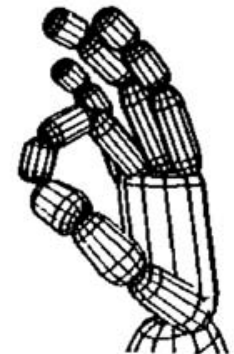

b

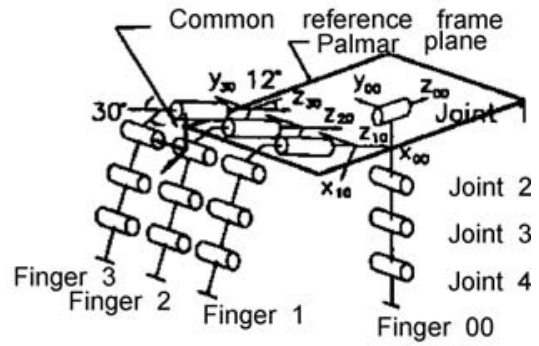

c

Fig. 1a-c. Various hand models

to the idea of task orientation [17] and naturalness of creases [11]. We are inspired by the concept of constraints from Yasumuro et al. [36] and the naturalness of the postures by Kuch [18] and by Kurihara \& Miyata [19].

We rely on observing motions, measuring anatomy and on anatomical literature as several authors do, but it strikes us that many of them $[6,10,27,29,36,37]$, even the most advanced modelers [1], are trapped in e.g., a simple hinge analogy for the visual most accessible phalanx motions, an analogy that is denied by literature, or authors apply collision detection $[21,28,29]$ while passive skin indention is an important expressive for natural interaction.

We present a model of the human hand that has natural motion and constraints by skeletal linkage through biomechanical true joints and with a skeletal driven skin. The skin geometry is deliberately simple as it only serves to judge and establish the biomechanics.

In search of natural motion, we discuss the biological linkage of the individual to its environment through human evolution and ontogenesis (the development of the individual) of the hands anatomy. We present our approach to modeling and give a description of anatomical nomenclature. We introduce tasks and discuss how constraining elementary tasks originate in the environmental linkage and give experiential descriptions. We discuss anatomical taxonomies and motions from literature. To evaluate our model we compose a taxonomy of elementary tasks that describe all hand motion in a confined two-dimensional motion parameter space. Then we discuss how joint surfaces and forces bring forward specific natural motions and show how their simulation has a biological basis. On this basis we model a natural joint axis (NJA). We discuss implementation of the NJA to the different joints and the effect on motion freedom. We discuss the constraints and how they bring down the number of action parameters in our natural human hand model (NHHM). At the end we model the skin and discuss the model description with joint hierarchy. We benchmark by fitting our skin-model with a common joint model and visually compare an elementary extension-flexion motion to the same motion of the NHHM with the NJA. We evaluate by visually comparing the taxonomy to the real hand. We conclude, give recommendations and future plans. In the appendix sequences of NHHM postures from different angles are given and can be compared to pictures of the real hand. The animated NHHM can be found on our website.

\section{Considerations}

In hand motion research many hypotheses "atomize" the perception-action coupling as if before catching a ball, we are able to decide separately about direction, distance, speed and deceleration of the ball and find the right posture of our body, arm, wrist and the hand's segments. Or are we? We think not, so we agree with Runeson: “... to what is biologically relevant ... the concepts of physics are often not very convenient." [30, pp. 172-173].

Biologically relevant is the linkage of man to his environment through evolution for the species and for the individual through the ontogenesis of its anatomical elements. In the linkage the anatomy has evolved and develops passive and dynamic constraints [34, pp. 505-507], in and around joints and over successive joints, to prevent energy loss and damage in tasks. The constraints cause motions and tasks to have a minimal amount of action parameters. As modelers we have the same interest, to find and model motion constraints cf. [36, p. 9].

\section{Approach}

The effects of ontogenesis and evolution cannot be separated i.e., an individual hand forms a whole, adapted to tasks explored from childhood on, resulting in specific constraints while in the same time it is a general example of tasks and constraints for the species. So for extracting general anatomical principles any hand can serve. As a statistical mean hand does not exist (is inherently unnatural), only personalized data can sustain general principles. Many data can be found $[5,33]$ but always depersonalized.

We capture personalized data from the skin and motion of the first author's right hand, the old-fashioned way, with calipers, caliper rulers, compasses, rulers and analyzed 


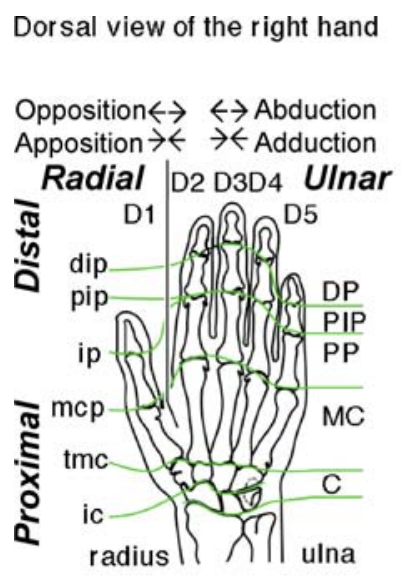

Motion

The thumb, index, ring finger and little finger adduct towards and abduct from the long finger. The four fingers oppose ulnary and appose radially, for the thumb it is the reverse. All fingers flex volary (towards the palm) and extend dorsally.

Fig. 2. Anatomical description

radiographs (taken of the thumb). We let eight subjects obtain data from their fingers joints to analyze the effect of joint surface asymmetries on motion. We then process all data from the skin and biomechanics into technical drawings of the skin mesh and a virtual mechanical model.

Now we suggest consulting Fig. 2 and the description of anatomical nomenclature. After that we discuss in detail elementary tasks and their constraints.

\section{Elementary tasks and constraints}

Most hand motion-research concerns tasks cf. [22, Ch. 2] like grasping objects and tool use (Fig. 3a-d). In these tasks the pressure on the skin deforms the tissue between skin surface and skeleton to "conform to the contours of objects" [34, p. 854] and, by sensing differences and continuous motor-control [22, p. 205-213], the contact area is dynamically optimized to the skin friction by which objects safely can be held.

Now holding a tool is a task that originates early in evolution, but we observe a larger variety of tasks, as "early" as holding, in which the fingers are the "objects" and the hand itself is a "tool" e.g., making a cup with all
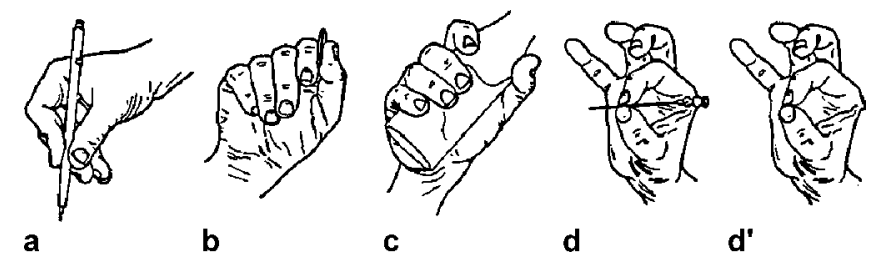

Fig. 3a-d. Tool use and the hand as tool (d') fingers to heave water and drink from it, also observed by Kapandji [16] [22, p. 30]. In this task the adducted segments optimize mutual skin contact areas to seal against passing water. Or it is a communicative "tool" with the task to express meaning and emotion e.g., in the O.K. posture the opposing forces optimize the skin indention of the fingers tips' to express the degree of appreciation (Fig. 3d').

In the next few paragraphs, we will address more elementary tasks and constraints, structural and functional, and show how they form a coherent biological whole.

Structural constraints. For their opposition and adduction tasks, the segments are structurally constrained, evolutionary and ontogenetically, depending their longitudinal and lateral hierarchy (Fig. 4).

Elementary tasks and constraints. We observe and discuss three elementary tasks; "Containing", "Getting" and "Holding". "Containing" is constrained in adduction and "Getting" in abduction, both active in extension-flexion and with their different starting and ending tasks. "Holding" comprises all oppositional and appositional tasks; in which adduction-abduction and flexion-extension actively cooperate and segments are constrained by themselves or by held objects.

- Containing motion. In the CUP the finger segments are actively constrained in adduction supported by the structural adducting orientation of the phalanges (Fig. 4b3). From the EMPTY posture (meaning: no containing, Fig. 5a3) the hand flexes via the CUP (Fig. 5b3) into a fully closed and constrained "box" with extended fingertips we call a CAGE (Figs. 4a3 and 5c3) and that is also observed by other hand modelers [18, $20,24]$. We suppose it is elementary as more than half of the world population eats insects and way back in evolution started to catch them without tools.

- Getting motion. The GRASP is a prehensile posture [26] that prepares the hand to hold objects as large as possible for that particular hand, the thumb takes

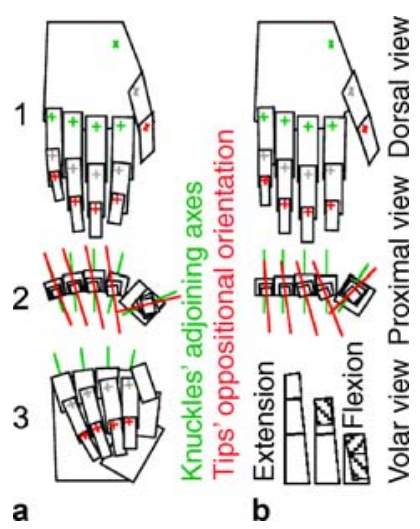

Fig. 4a,b. Structural orientation 


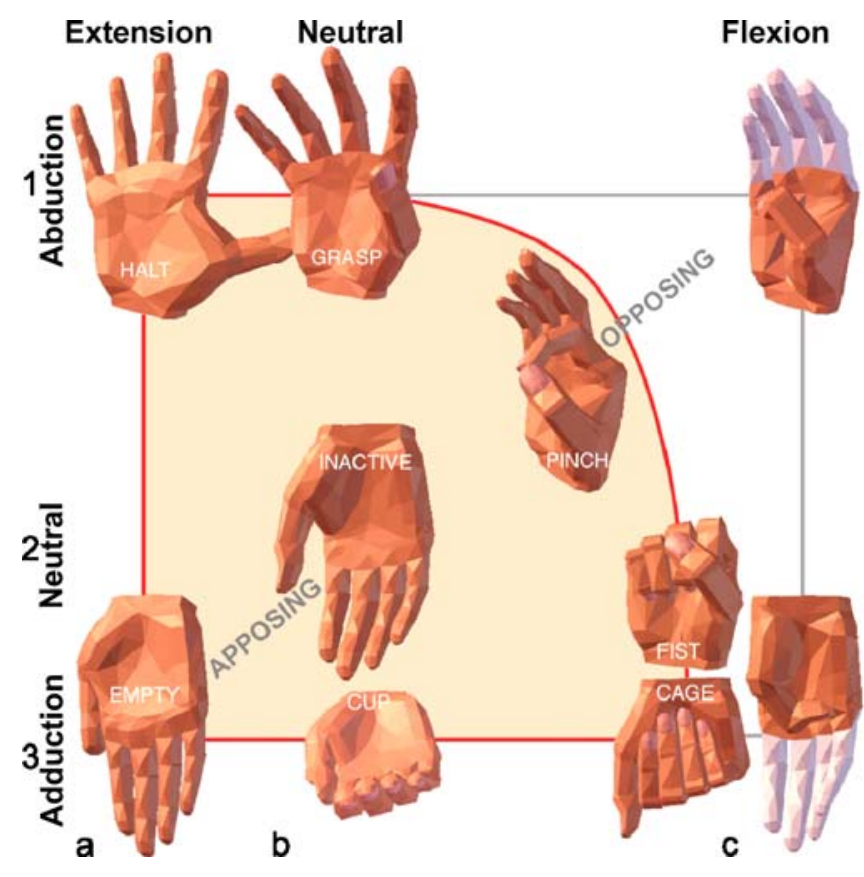

Fig. 5a-c. Taxonomy and motion space

a pose analogous to the fingers; they roughly ascribe a sphere with all articulate segments between flexion and extension actively constrained in abduction. Getting begins (or ends) with the extended and $a b-$ ducted HALT posture (meaning: no action, Fig. 5a1/2), it flexes along abduction constraints into the GRASP (Fig. 5b1) ending in a FIST (Fig. 5c3). The fist's dynamics withstand expected or sensed external forces (Sect. 5.2), it is a weapon for defense and attack and as a tool on the raised under arm it conveys meaning, i.e., protestation.

- Holding motions: The INACTIVE hand (Fig. 5b2) is a natural starting position for hand-motion when it hangs from the inactive arm: with all segments in a natural neutral position and none in the same plane (Fig. 4a1-2), see also Cooney et al. [7, p. 1378] and Kurihara \& Miyata [19, p. 356]. Note that it is variable as its shape depends on gravity: on the vertically raised under arm it will be closer to that of the fist and that it is quite different from the forced flat posture (Fig. 4b1) that some investigators start from although segment axes then also are not in one plane and motion axes are not parallel or perpendicular to that plane (Fig. 4b2).

In the holding motions the opposition of thumb and fingers grants the dynamic stability that allows for elongation of the underarm with tools: objects for inspection, attack or defense e.g., a stick for probing, a bottle to drink from (Fig. 3c), a hammer or a weapon. The thumb and fingers' opposing motions complement each other, sustained by the structural opposing orientation of the phalange segments (Fig. 4a2), and so the thumb differs from the fingers only by having larger motion freedom. In PINCHES (Figs. 3d,d', 5b/c1/2 and appendix) the thumb and the involved finger oppose to their abduction constraints. The thumb-index pinch serves precision holding (Fig. 3d) and is probably an evolutionary function to study small life form e.g., insects, plant parts, etc.

\section{Taxonomy and motion space}

In hand motion-research many taxonomies can be found and they are infinite. Thus, when a new task is found it is added to an existing taxonomy cf. Cutkoski \& Howe in [22, p. 26]. To evaluate our hand model, we discuss why and how constrained elementary tasks can be arranged around the inactive hand to describe all hand motion in a simple and confined taxonomy.

Moving from INACTIVE to CUP, GRASP and PINCH and vice versa, we experience very little effort. When flexing from a CUP the apposed hand can "do" a CAGE very fast, but making a FIST is uneasy and slow. From GRASP the abducted hand easily flexes into a FIST, while it is uncomfortable and slow to do a CAGE.

We like to share this with our readers as we infer and experience that the containing and the getting motion are complementary: in containing, task-directed forces are actively constrained in adduction while extension-flexion only serves motion for adaptive postural transition. In getting, the flexing forces are task-directed while adaptive postural transition is constrained in abduction. The two motions also have an complementary meaning: containing serves conservation as it generates life-sustaining tools, getting serves action, as it is life exploring and defending. The holding motions serve conservation and action.

All the elementary tasks find their place in a confined motion space on two anatomical motion dimensions: adduction-abduction and extension-flexion (Fig. 5). Central to all motion is INACTIVE on the neutral position of both dimensions and the apposition-opposition diagonal. Getting and containing bound the field of Holding motions. Pinches are at the boundary of the getting motion, on the apposition-opposition diagonal.

For clarity the thumb in full flexion is positioned outside the Holding field. The thumb has two constrained end postures, in full abduction and opposition the tip of DP touches the palm at the volar side of the little fingers' mcp (Fig. 5c1) and in full adduction the phalanges appose to the palm (Fig. 5c3). In the latter thumb position the integrity of its joints and bones are threatened in the "false" fist (see appendix), as the fingers immobilize the thumbs phalanges the dynamical adaptation of the joints (Sect. 5.2) is hampered. 


\section{Anatomy and natural motions}

We discuss joint articulate surface shapes (JASS), muscle forces and outside forces that together cause three main types of segment motion. We discuss the possibility of rotational axes, dynamical adaptation and describe and test the effects the JASS have on motion.

\subsection{Natural motions and forces}

We distinguish passive, loaded and unloaded motion.

- In passive motion the antagonist muscle system is inactive so only the geometry of the JASS and surrounding tissue restrict the involved segments while they are exposed to forces from outside, whether gravity or palping by a diagnosing surgeon. Kapandji [16] and Cooney et al. [7, p. 1371] conclude that tmc has two active axes but the bone on it can be passively moved. As JASS geometries themselves are not modeled, passive motion is not included in this publication.

- In loaded motion antagonist muscle forces put into motion anatomical masses and/or loads from objects, dynamically bringing about decelerations and accelerations during action e.g., swinging a hammer or swaying forward a leg while walking [34, p. 505]. This results in segment motions, during which the JASs have ample contact (anatomists, rather misleadingly, call these 'adjunct motions'). These very complex motions are the real natural motions, but as we do not model gravity, loaded motion is beyond the scope of this article.

- In unloaded motion ("unconstrained" as Tsang et al. call it [32]), so without loads from objects and segment masses, only the joint surface friction and antagonist muscle forces are balanced and segment motions coerced by the JASS. These unloaded motions are modeled.

\subsection{Joint surfaces, axes and motion}

Through flexion-extension JASS are asymmetrical; there is no clearly positioned joint axis. Some modelers and anatomists therefore model 'mean' axes e.g., [7, 20, 29] and thereby compromise natural motion. We discuss how cooperation of the proximal male (convex) and the distal female (concave) shapes of the cartilage clad bone endings coerce natural and, in the same time, rotational segment motion.

In all joints the JASS are asymmetrical during flexionextension causing conjunct motion and ovoid motion. In mcp and cmc (tmc) JASS are ellipsoid for the adductionabduction motion. We discuss and test these JASS properties and will show that rotational axes can very closely simulate the segment motions on all JASS.

- All JASS cause conjunct motion. Hollister et al. [13] establish tmc's "biaxiality" (the reason for hyphenat- ing is given in the conclusion to Chap. 5) by using an ingenious mechanical apparatus, an 'axis-finder', and prove that there is "one (rotational axis) in the trapezium and one in the metacarpal. Axes that do not cross segment axes and that are neither perpendicular to those nor to each other." As we are dealing with the non-Cartesian nature of biology, we can safely assume that in all joints, motion axes are non-perpendicular to segment axes causing conjunct rotations [34, pp. 499$500]$ i.e., rotations of the segment axis that are coupled to the flexion extension motion (Fig. 6a, conf. Fig. 6b, oblique ulnar view, distal is under). These motions of the segment axis and the structural segment orientations of IP and DIP keep the fingers adducted through extension-flexion in the containing motion and enhance stability in getting and holding [34, p. 507].

- All JASS have "ovoid" shaped volar-dorsal sections (Fig. 7a,a'), and together with conical motion they determine the flexion-extension motion. Coincidence of the congruent [34] ovoid surfaces is called the "closepacked" position (Fig. 7a), joints lock at the end of the motion range to avoid instability. Just before joints lock, a sense-muscle loop prevents them from close packing [34, p. 505, 507]; they are dynamically constrained e.g., enabling a joint to conserve energy as in the knee while standing or enabling the joints in

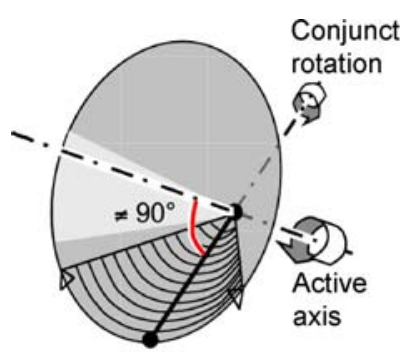

a Conical segment motion

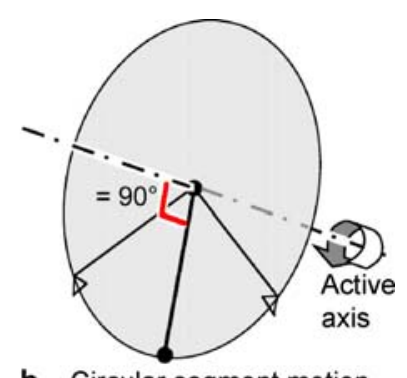

b Circular segment motion
Fig. 6a,b. Conical and circular motion
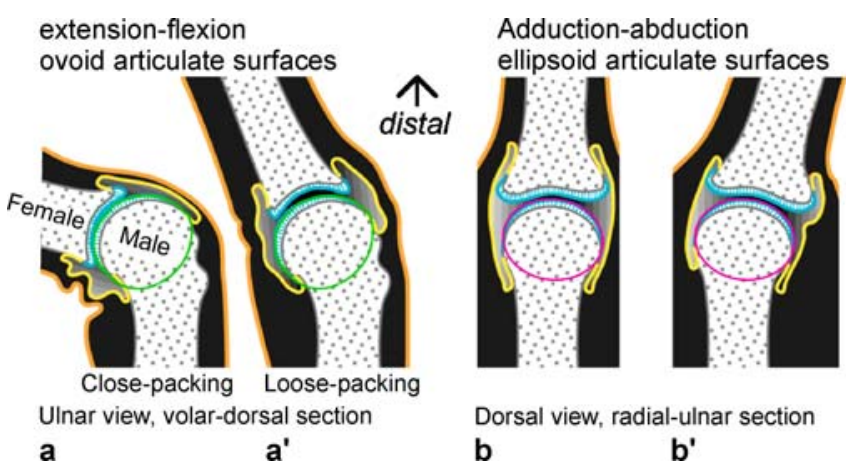

Fig. 7a,b. Ovoid and ellipsoid joint articulate surfaces 
the fist to maintain structural integrity as the organism anticipates expected forces and adapts to exerted forces.

The ample space between not coinciding JASS, the loose-packed position (Fig. 7a'), contributes to the low friction that is essential to the motion of anatomical joints [34, p. 503]. Ovoid motion increases stability in grasps [22, p. 263].

In "Grays"” [34, p. 507] the effect of conical and ovoid motion together is described with plasticity: “ . . . joints are screwed home...".

- Mcp and cmc joints have ellipsoid surfaces i.e., elliptical radial-ulnar sections and about volar-dorsal in tmc (Fig. 7b,b') and those surfaces determine the adduction-abduction motions on them.

\subsection{Measuring motions}

We first measure adduction-abduction of the tmc JASS by taking radiographs and analyze them with a compass and ruler comparably to Buchholz et al. [5] and other authors. We only derive the distance between tmc axes from this method $(18.4 \mathrm{~mm}$, after compensation for plate distance and radiation spread).

We did not further apply this capturing and analyzing method as making, sharp enough, in vivo radiographs of the fingers' mcp ovoids is, for us, technically impossible and the classical analysis method is prone to even larger errors if applied to even smaller joints.

By analyzing segment motions on ovoid and ellipsoid JASS with compass and ruler, we devise a method that is more reliable than analyzing joint articulate surfaces, let alone bone articulate surfaces. We validate the results of our analysis by graphic simulation.

- We measure ovoid motion by having eight subjects draw extension-flexion of D2, 3 mcp (with fixed MC) and D2, 3 pip (with fixed PP). To draw we devise clamps to the respective phalanges with pen-holders perpendicular to the segment axis and the pen about parallel to the flexion-extension axis. Drawn ovoids are analyzed in the old-fashioned way with compass and ruler (Fig. 8a) but now 20 to 30 -fold larger than

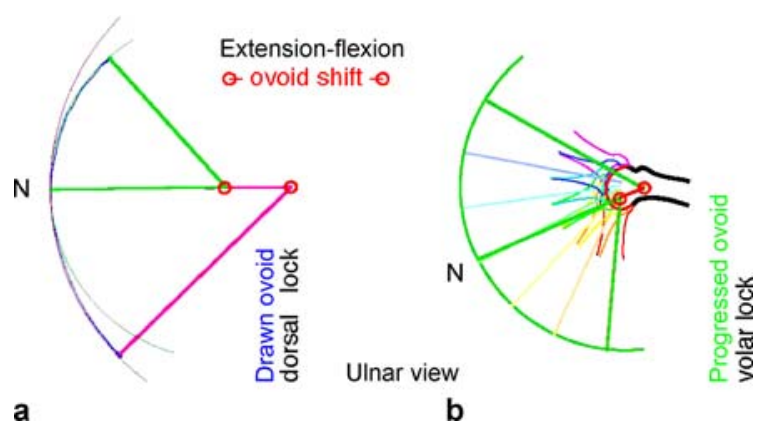

Fig. 8a,b. Measuring/progressing ovoid motion

in former measurements enabling collecting precise data of ovoid motion. And we two-dimensionally simulate ovoid motion by progressing ovoid sections on each other (Fig. 8b). From measuring we conclude that all ip ovoids lock dorsally, confirming literature [34, p. 509] and mcps have varying locking positions in and between subjects (SS; MS = male, FS = female), while the literature gives a volar locking position [34, p. 509], see Table 1. An et al. discuss [2] that mops have to be volarly constrained to prevent destabilizing the joints from intersection of segment axis and adduction-abduction axis. From the variation in individual locking positions, we assume the volar constraining of mcp described by An et al. is independent from the ovoid locking safety system. From our analysis of SS drawings (first author: MS3) and the progressions (Fig. 8), we conclude that natural ovoid motion is defined by two separate circle-segments with different radii that change at the NEUTRAL position of its segments (as in INACTIVE) and are modeled with two clear rotational axes.

- We test ellipsoids by progressing elliptical sections on each other and measure as we did with the ovoid sections, now with the pen about parallel to the assumed adduction-abduction axis. We conclude, somewhat to our surprise, that the resulting motion pattern is circular (Fig. 6b) but infer that a circular JASS cannot exist in biology as it will have no low friction positions (can never be "loose packed").

Table 1.

\begin{tabular}{|c|c|c|c|c|c|c|c|c|}
\hline \multicolumn{9}{|c|}{ OVOID shift, testresults in $\mathrm{mm}, \mathrm{d} .=$ dorsal lock, $\mathrm{v} .=$ volar lock } \\
\hline SS & FS1 & FS2 & FS3 & FS4 & MS1 & MS2 & MS3 & MS4 \\
\hline L.D3 & 98 & 102 & 104 & 105 & 106 & 114 & 116 & 127 \\
\hline D2 mcp & d. 5.0 & v. 11.5 & d. 3.5 & v. 4.5 & d. 6.0 & v. 15.0 & d. 4.5 & d. 10.8 \\
\hline D2 pip & d. 6.5 & d. 5.2 & d. 6.0 & d. 7.5 & d. 5.0 & d. 6.9 & d. 5.0 & d. 9.9 \\
\hline D3 mcp & v. $0-18.5$ & v. 18.4 & v. 4.5 & d. 5.5 & v. 6.4 & v. 21.0 & d. 5.6 & d. 11.2 \\
\hline D3 pip & v. 9.0 & d. 14.7 & d. 7.0 & d. 3.5 & d. 5.0 & d. 9.3 & d. 6.3 & d. 11.0 \\
\hline
\end{tabular}


Adduction-abduction can be modeled with one clear rotational axis.

So at any given point in time motions on mcp and cmc (tmc) are simulated by two rotational axes, but we need to define three rotational axes as one of them shifts.

\section{Modeling natural motion}

Based on discussions and findings, we discuss here a natural joint axis (NJA) and how the four different types of joints in the human hand can be modeled with the NJA. We discuss how the segments on NJA describe conical, ovoid and circular motion and are structurally oriented. We discuss the constraints and how they bring down the number of action parameters. We end with a description of the skin and its motion.

The joints have a local, right-handed reference system; the modeled segments have local space references, both illustrated in Fig. 9.

\subsection{Natural joint axis}

We compose a natural joint axis (NJA) with three local coordinate systems (three LCS or Euler angles, Fig. 10, distal is to the left). LCS-1 (green) is static: it is set with the offset from the proximal NJA and, with respect to the proximal segment axis, the structural orientation angle of this NJA and the neutral orientation angle of the INACTIVE

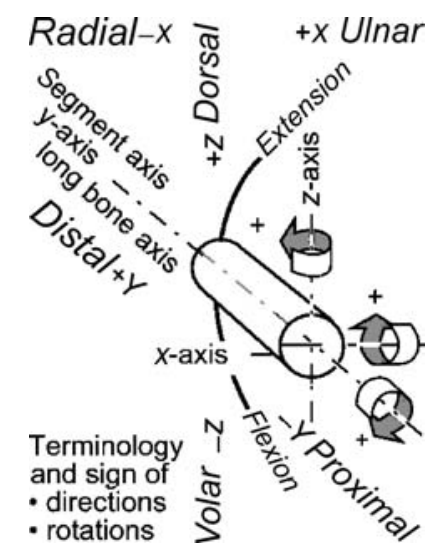

Fig. 9. Modeling references
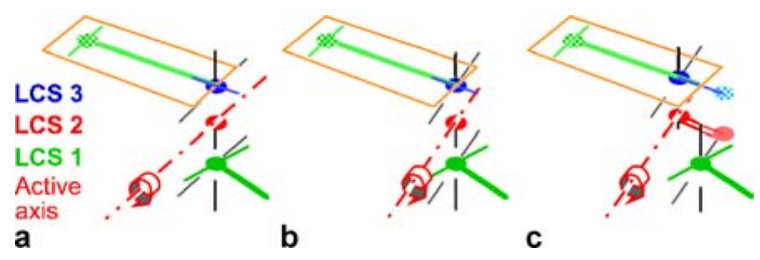

Fig. 10a-c. Natural joint axis posture. LCS-2 (red) is dynamic, modeled here are: the ovoid change or shift at the neutral position ( $y$-translation, Fig. 10c) and the interactive rotation ( $x$ or $z$ ). LCS-3 (blue) is again static: set is the angle between the active axis and the distal segment axis; whether the angle is $0\left(=90^{\circ}\right.$, Fig. 10a) to model circular motion or the angle is not 0 $\left(\neq 90^{\circ}\right.$, Fig. $\left.10 \mathrm{~b}\right)$ to model conical motion ( $y$-co-rotation).

The transformation matrix for the skin geometry is, if the joint locks volarly (flexes with a small radius) symbolized with:

$$
\begin{aligned}
\mathbf{M}_{\mathrm{NJA}_{\text {ext }}=} & \mathbf{R}_{\text {distal }} \bullet \mathbf{T}_{\frac{1}{2} \text { ovoidshift }} \bullet \mathbf{R}_{\text {interactive }} \bullet \mathbf{T}_{-\frac{1}{2} \text { ovoidshift }} \\
& \bullet \mathbf{R}_{\text {neutral }} \bullet \mathbf{R}_{\text {proximal }} \bullet \mathbf{T}_{\text {offset }} \\
\mathbf{M}_{\mathrm{NJA}_{\text {flex }}=} & \mathbf{R}_{\text {distal }} \bullet \mathbf{T}_{-\frac{1}{2} \text { ovoidshift }} \bullet \mathbf{R}_{\text {interactive }} \bullet \mathbf{T}_{\frac{1}{2} \text { ovoidshift }} \\
& \bullet \mathbf{R}_{\text {neutral }} \bullet \mathbf{R}_{\text {proximal }} \bullet \mathbf{T}_{\text {offset }}
\end{aligned}
$$

(If the joint locks dorsally $\mathbf{T}_{\text {ovoidshift }}$ 's have to change sign.)

In Fig. 10c the NJA is set as an $x$-axis with conical and ovoid motion ( $y$-shift to small radius). Settings of LCS 1 and 3 are calculated on beforehand with structural and neutral orientation as single summation, if joint configurations do not require setting (are 0), no calculations are carried out: the composition of NJA is variable, and by that it can be applied to simulate all four types of joints in the human hand.

\subsection{Joints and motions}

In the human hand four types of joints are distinguished: ip, mcp, cmc and ic. Their different JASS cause specific segment motions, we will discuss in four paragraphs how the four different joints are modeled with NJA and bring forth different types of motion.

We explicate in Table 2 what kind of DOF the four types of joints have, in the column "Motions" they are summated for the whole hand, but before applying the constraints (in Table 3 after constraints). We would like to compare our DOF e.g., with Kurihara and Miyata [19, p. 356], but as as they do not explicate their DOF this is impossible.

- The ip, pip and dip joints that only flex and extend are described as bi-condylar: having double, ovoid JASS (Fig. 11a, oblique dorsal view and with distal on the left, as in Figs. 12 and 13), the complementing groove and ridge in the proximal and distal surfaces disable radial-ulnar motions of the segments, sustained by ligaments. The flexion-extension segment motion has $y$-co-rotation and $y$-shift. The joints lock in full extension (Fig. 11c black arrow). They are modeled with one NJA (Fig. 11b). On nine ips, 27 segment motions are possible (Table 2).

- The mcp has ovoid-ellipsoid JASS. The radial-ulnar elliptical JASS coerce circular adduction-abduction 
Table 2.

\begin{tabular}{|c|c|c|c|c|c|c|c|c|c|c|c|}
\hline \multirow{2}{*}{$\begin{array}{l}\text { Joint } \\
\text { name }\end{array}$} & \multirow{2}{*}{$\begin{array}{l}\text { Axis type } \\
\text { in hierarchy }\end{array}$} & \multicolumn{3}{|c|}{$x$-axis } & \multicolumn{3}{|c|}{$z$-axis } & \multicolumn{4}{|c|}{ Joint and motions } \\
\hline & & $\begin{array}{l}\text { Free } \\
\text { axis }\end{array}$ & $\begin{array}{l}y \text {-coro- } \\
\text { tation }\end{array}$ & $\begin{array}{c}\text { Coerced } \\
y \text {-shift }\end{array}$ & $\begin{array}{l}\text { Free } \\
\text { axis }\end{array}$ & $\begin{array}{l}y \text {-coro- } \\
\text { tation }\end{array}$ & $\begin{array}{c}\text { Coerced } \\
y \text {-shift }\end{array}$ & Joints & $\begin{array}{l}\text { Motion } \\
\text { axes }\end{array}$ & $\begin{array}{l}\text { Coerced } \\
\text { motions }\end{array}$ & Motions \\
\hline ip & $x$ & $\emptyset$ & o & o & & & & 9 & 9 & +18 & $=27$ \\
\hline mcp & $z)(x$ & $\emptyset$ & o & o & $\varnothing$ & & & 5 & 10 & +10 & $=20$ \\
\hline $\mathrm{cmc}$ & $x)(z$ & $\emptyset$ & $\circ$ & $\circ$ & $\varnothing$ & o & & 5 & 10 & +15 & $=25$ \\
\hline$i c^{*}$ & $x)(z, z)(x$ & $\emptyset$ & $\circ$ & $\circ$ & $\varnothing$ & $\circ$ & $\circ$ & 12 & 24 & +48 & $=72$ \\
\hline & & & & & & & & 31 & 53 & 91 & 144 \\
\hline
\end{tabular}

* Hypothetical

Table 3.

\section{Constraints}

\begin{aligned} & \hline 53 Motion axes \\ & $53-24=29$ Proximal cmcs \\ & $29-3=26$ D2, 3 simplified cmcs \\ & $26-1=25$ D4 simplified cmc \\ & $25-1=24$ D1 tmc2 unconditional by D1 mcp1 \\ & $24-1=23$ D2, 3 cmcs unconditional by D1 tmc1 \\ & $23-1=22$ D4 cmc unconditional by D5 cmc \\ & $22-2=20$ D5 cmc 1, 2 unconditional by D5 mcm 1, \\ & 20 is $38 \%$ Free axes left \\ & $20-8=12$ D2, 3, 4, 5 conditional dip-pip-mcp \\ & 12 is $23 \%$ Free axes left \\ & \hline\end{aligned}

motion; the volar-dorsal ovoid JASS coerce ovoid (Fig. 12a) and conical (Fig. 12c blue-green) segment motion in flexion-extension. Over flexion segments show gradually smaller adduction-abduction rotations (Fig. 12c red motion circles), the cones described by the segments have varying apex angles; from a small angle in flexion to a large angle in extension, in the thumb it extends further to a 'negative' large angle. In flexion the joints' motion is probably constrained by ligament tissue in the modeled extension by ovoid locking, (Fig. 12c black line/grey arrow).

The proximal $z$-axis (Fig. 12b red) has circular motion, variably constrained by the distal $x$-axis that also has $y$-co-rotation and $y$-shift (Fig. 12c ice-blue). The $z-x$ axes order determines the segment motion pattern. The offset between $z$ and $x$ is zero. The $z$-axis and the $x$-axis each are modeled with NJA. On five mops the segments can perform 20 motions.

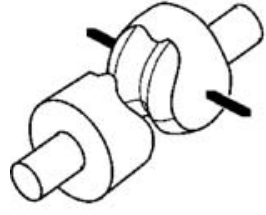

a

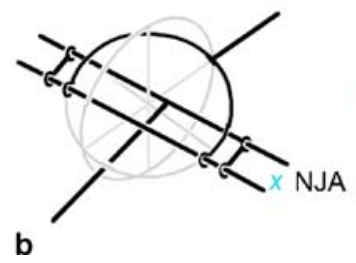

c

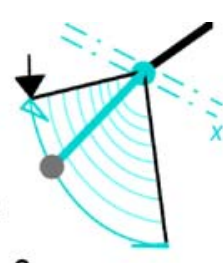

c

.

$a$

- Tmc, the cmc of the thumb, is described having a saddle (or sellar) surface (Fig. 13a1) and by Cooney et al. [7] and Hollister et al. [13] as being biaxial. The metacarpal describes a quarter of a cone with small apex angle and ovoid motion (Fig. 13a2) on the proximal $x$-axis that locks in flexion (Fig. 13b2 blue-green, black arrow). On the distal $z$-axis the metacarpal is adducted-abducted and describes a cone with a large apex angle (Fig. 13b2 pink). Independently the two axes enable the metacarpal of the thumb to circumscribe the metacarpal of the index, the proximal an $x$-axis with $y$-co-rotation and $y$-shift, the distal a $z$-axis with $y$-co-rotation. The $x-z$ axes order and their offset cause the typical different segment motions on tmc

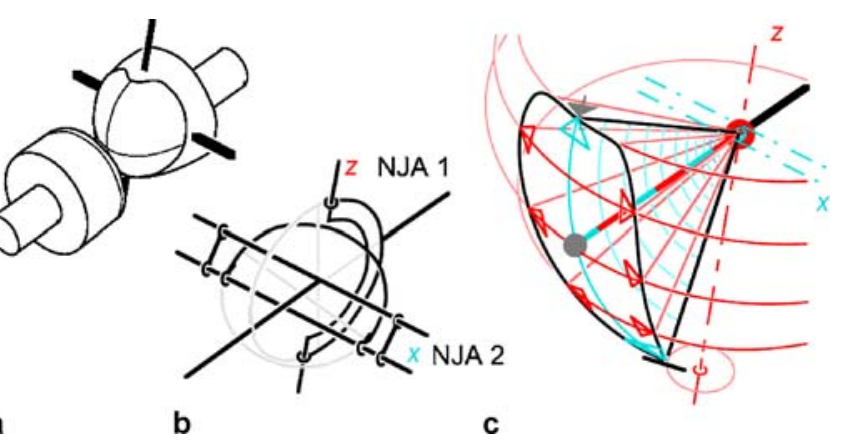

Fig. 12a-c. Modeling mcp (for volar lock)

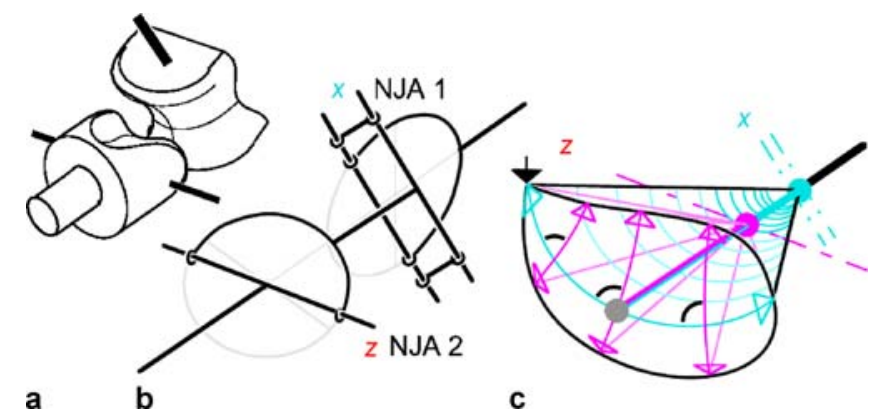

Fig. 13a-c. Modeling tmc/cmc (with volar lock)

Fig. 11a-c. Modeling ip, pip and dip (for dorsal lock) 
and mcp. The cmcs of the fingers are described as having 'complex sellar' JASS [34, p. 657], but we observe no motions more complex than those of tmc. The $x$ axis and the $z$-axis are modeled with the NJA. On five cmcs, there are twenty-five segment motions.

- The ics are described as having 'compound sellar' JASS [34, p. 651]. Several authors [20, 24, 27] argue that ics motions are small. We observe from anatomical drawings that the JASS have a flatter curvature than cmcs have, so axes are further apart i.e., motions can be large but based on very small rotation angles. We assume their compound character to be bi-axial with $y$-co-rotation and $y$-shift on both axes: hypothetically between eight carpals twelve biaxial ics can be modeled each with two NJA; and the number of segment motions will be seventy-two (See Table 2).

We summate that a 144 segment motions characterize natural motion of the human hand. Without constraints this is a hard task to handle for any human or modeler. So next we will discuss how these motions are constrained i.e., how we remove all un-free motions to end with the free axes; the action parameters.

\subsection{Constraints}

We distinguish passive, coerced, mobility and tendon constraints. First discussed are the passive constraints that do not bring down the number of free axes but prevent unnatural motions in animation. Then we discuss motions that are constrained by coercion of the JASS. Next we discuss the mobility constraints; very small motions are removed or combined in single or simplified NJA. Last we discuss the constraining by tendons and tendon combinations in which we distinguish unconditional and habitual constraints. We summarize these in Table 3 (see also Fig. 15).

Passive constraints. On all axes end-of-ranges model the constraining tissues around joints. In extension-flexion we model end-of-ranges just before joints lock, we do not model bi-lateral range constraints between the fingers mcps as they are dynamic. In mcps adduction-abduction the $z$-axis end-of-range depends on the $x$-axis angle of rotation; for which we model a Catmull-Rom spline with five control points on the radial and the ulnar side.

Coerced constraints. The JASS coerce 91 conical and ovoid motions modeled with NJA so from hundred and forty-four motions we have 53 axes left.

Mobility constraints. We remove all ic and cme motions that we inferred from the anatomical structure but do not observe. The, still, observed very small motions of the joints involved are modeled by simplifying and combining NJA.

- Inter carpal axes. The ic have 24 axes that bridge motions of the wrist and the cmc. Near the motion extremes of the wrist the influence of the ics to the motion of the little finger is considerable [31] and to the other fingers noticeable. We remove the ics and model the effect of carpal motion by situating cmcs more proximal than they anatomically are. So 29 axes are left.

- Carpo meta-carpal axes. D1 tmc is fully modeled, D2 and D3 cmcs are so immobile with respect to each other that we model them with one NJA; from four axes one remains. As D4 $\mathrm{cmc}$ has a rather immobile role in folding the palm it is modeled with one NJA instead of two, D5 cmc is fully modeled like D1 tmc, so 25 axes are left.

\section{Tendon constraints}

- Unconditional constrained motions. Tendons and muscles around the cmc axes enable folding the palm. In D1, seven to eight tendons over tmc and mcp constrain motion: tmc- $z$ depends fully on mcp- $z$ leaving us with 24 axes. In D23 the $\mathrm{cmc} z$-axis depends fully on D1tmc- $x$, so now 23 axes are left. D4 cmc- $z$ depends on D5cmc- $x$ leaving us with 22 axes. And D5 cmc- $x-z$ depend on D5cmc- $x-z$ so we have 20 axes left. Now D1cmc- $x-z$ conditions D5 cmc- $x$ to fold the palm but as both depend on their respective mcps they do not reduce the number of axes. We observe that the palm folds by pivoting around D4mcp: D2-3cmc rotates dorsally/ulnary and D5cmc volarly/radially. So 20 axes, one in tmc, ten in the mcps and nine in the ips move the hand and will animate the NHHM. This reduces the number of action parameters to $14 \%$ of the total number of motions.

- Habitual constrained motions. To our observation habitual tendon constraints over mcp-pip-dip in the containing motion differs from those in the Getting motion. In the containing motions the four fingers are constrained between mcp and pip while dip is not constrained (is passive). In the getting motion mcps are less flexed and ips flex further until the fingers' tips indent the palm in a row, between their proper metacarpal and the metacarpal radially next to it. As all, very different, digits end in line, we conclude that in every individual hand there is a relation between phalange length and habitual tendon constraints. We did not establish this relation, but the observation does not sustain generic habitual motion relations between dip and pip as suggested by the sometimes quoted, linear $0.66 \mathrm{dip} /$ pip relations $[27,30]$. In the modeled hand, the thumb is independent. The ips of the four fingers are constrained by mcp- $x$ and, if constraints agree with animation, only the 12 active axes of D1tmc- $x$, D1ip and D2-5 mcp- $x$ and $z$ have to be handled; a reduction of action parameters to eight percent of the total number of motions.

\subsection{Modeling the skin}

The skin is a protective non-elastic but flexible organ that, under its plies and creases, is anchored with fascial ligaments to underlying structures $[34$, p. 662,854$]$. Fascial 
ligaments withstand the shear and stress caused by friction and conformation to shapes. The heavier fascial ligaments cause the large and deep plies near the joints. Small ligaments cause the creases that are more evenly spread, with high spatial frequency in the phalanges and the volar side of the palm, and low frequency dorsally in the palm.

Parts of the skin with a high spatial frequency of small fascial ligaments are modeled with static triangular faces (Fig. 15 green), skin parts with a low frequency are modeled with faces that have variable side-lengths (Fig. 15 blue). Each phalange model is distally open to accommodate the barrel shaped proximal end of the next phalange (Fig. 15a), together they model the dorsal protrusion and the volar crease and allow for all structural and motion changes at the joint. On the palm static and dynamic faces connect mostly at creases (Fig. 15 red).

The geometry is designed to have just enough faces to suit our observational evaluation. We keep the number of faces low with a variable mesh: the angle between vector-normal and face sides is kept about constant; vector density is highest in the fingers tips and nails. To keep

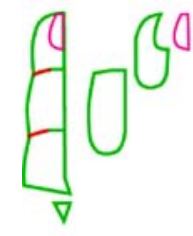

Ulnar view

a

Fig. 14a-c. Modeling the skin

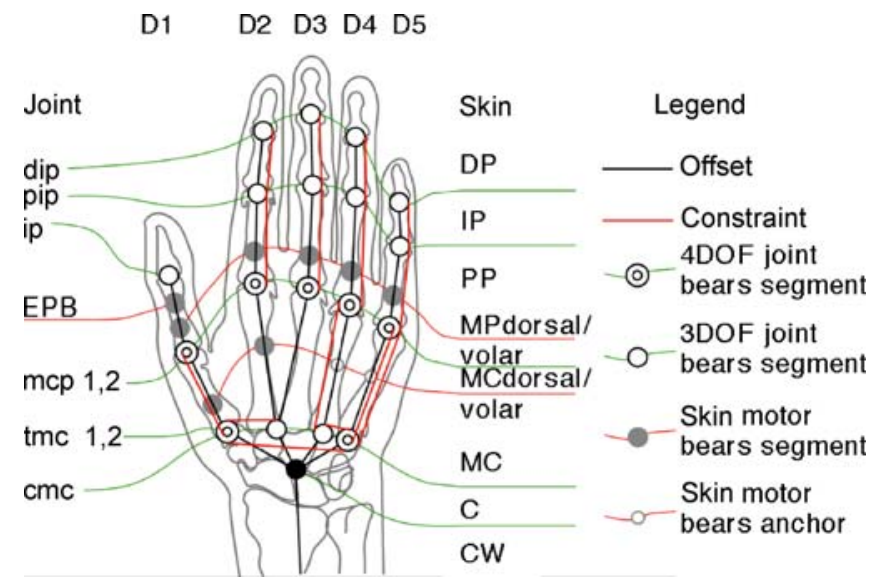

Fig. 15. Model description the number of segments low we model five D3 segments that are copied to scale the 15 segments of D2, 4 and 5 (Fig. 15bc light green), the nails (Fig. 14 pink) are different in all fingers and adapted accordingly. Fifteen segments model skin indention and bulging around the palm (including three single vectors, Fig. 14b red dots). The 40 segments have on average twenty-five static faces. In total about a 1,000 static faces and 250 dynamical faces, together 1,250 faces model the skin of the NHHM.

\subsection{Model description}

The NHHM's joint hierarchy (in Fig. 15, reference is to mcp, cmc and ic $x-z$ or $z-x$ joint configurations and to ip, pip, and $\operatorname{dip} x$ or $z$ configurations) shows the segmentlinked joint order and the constraints. Twenty-five NJA model motions of joints and the offsets between NJA model distances between joint axes. The skin geometry zero-vectors are attached to their proper proximal joints. The dynamical faces are connected to the static faces through vector transposition. To model active skin indention and bulging, eleven NJA, serving as skin-motors, have offsets and constraining couplings to the joint simulating NJA that are considered responsible for the skins motion. Passive skin indention is not modeled, but as it is an expressive feature in animation, games, etc., indentions can be simulated in animations, etc. by slightly crossing the skin on colliding segments; therefore, we deliberately did not model collision detection (besides that, visible or invisible: all substance indents under pressure). In the NHHM 35 NJA and 15 constraints model natural motion.

\section{Evaluation}

We visualize the NHHM on a computer screen against a background with natural texture and perspective, with next to it an interactive interface that shows the action parameters and NJA- and constraint tables. The NHHM is evaluated by visually comparing it to the real hand by 'negative-space' matching [8] of the real hand's boundaries with those of the NHHM.

We use the taxonomy while iterating between assessing postures and value setting the NJA's and the constraints. We start by comparing adduction in INACTIVE then go to flexions-extensions in the CONTAINING and the GETTING motion. Comparing abduction in the pinches and adduction in the thumb is done last. And, as all these motions cohere in a natural hand, we repeat this cycle several times.

In the appendix, postures of the NHHM can be compared in five sequences of radial-ulnar rotation $\left( \pm 39^{\circ}\right.$ steps). We show the "false" fist, the fully flexed and abducted thumb, folding of the palm with the thumb to little finger PINCH and the V-for-victory sign that can be compared to pictures of the real hand. 


\section{Benchmarking}

In our benchmarking, illustrated in Fig. 16, the motion effects of joint axes with single LCSs (Fig. 161) can be visually compared to joint axes fitted with the NJA (Fig. 162). We take the containing motion represented by the EMPTY (Fig. 16a), INACTIVE (Fig. 16b) and CAGE (Fig. 16c) postures. Only four action parameters, D2-5 mcp extensionflexion axes, are handled and cmcs and constraints are set according to the NHHM in both. In Fig. 161 the single LCSs are set for most natural results in Fig. 16a and Fig. 16b; but they end very unnaturally in Fig. 16c. The NHHM with NJA is shown in Fig. 162 that, together, represent its natural containing motion.

\section{Conclusions}

Our approach enabled us to present a segment-linked hand model with natural unloaded motion, the natural human hand model (NHHM). To be able to do so we devised a confined hand motion taxonomy and a natural joint axis (NJA) with constraints. The model was fitted with NJA and a simple skin geometry and we used the taxonomy to evaluate the biomechanics of our model. We did not devise a method by which it can be adapted to any individual hand.

- We made a contribution to natural hand modeling by bringing (back) into discussion the biological basis to it: the origin, natural aim and anatomy of the human hand.

- We made a contribution to natural hand modeling with a description of a confined and simple hand motion taxonomy.
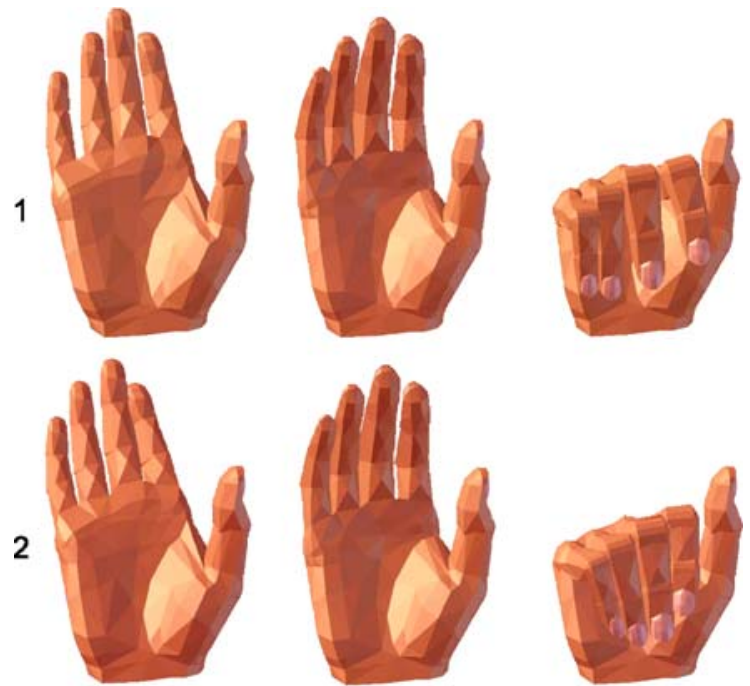

a
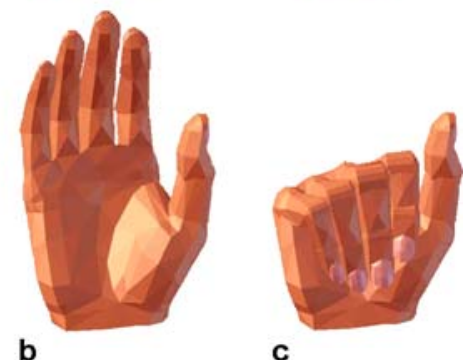

Fig. 16a-c. Single LCS (1) compared to NJA (2)
- We made a contribution to natural hand modeling by devising a natural joint axis that models cmc, mcp and ip joints in the human hand with natural motion and hypothesize how ic motions can be modeled.

- We made a contribution to natural hand modeling by showing that analyzing and applying constraints can reduce action parameters to twenty or even twelve of a hundred and forty-four different hand motions of our NHHM.

Animations of the NHHM can be seen via the internet (see appendix for URLs). The detailed joint hierarchy, joint variables, constraint variables and the skin geometries static segment list, static segment scaling variables and dynamic face list are, for non-commercial purposes, available from the first author.

The first two authors thank their co-authors and especially Prof. Dr. P.J. Stappers at Delft Technical University
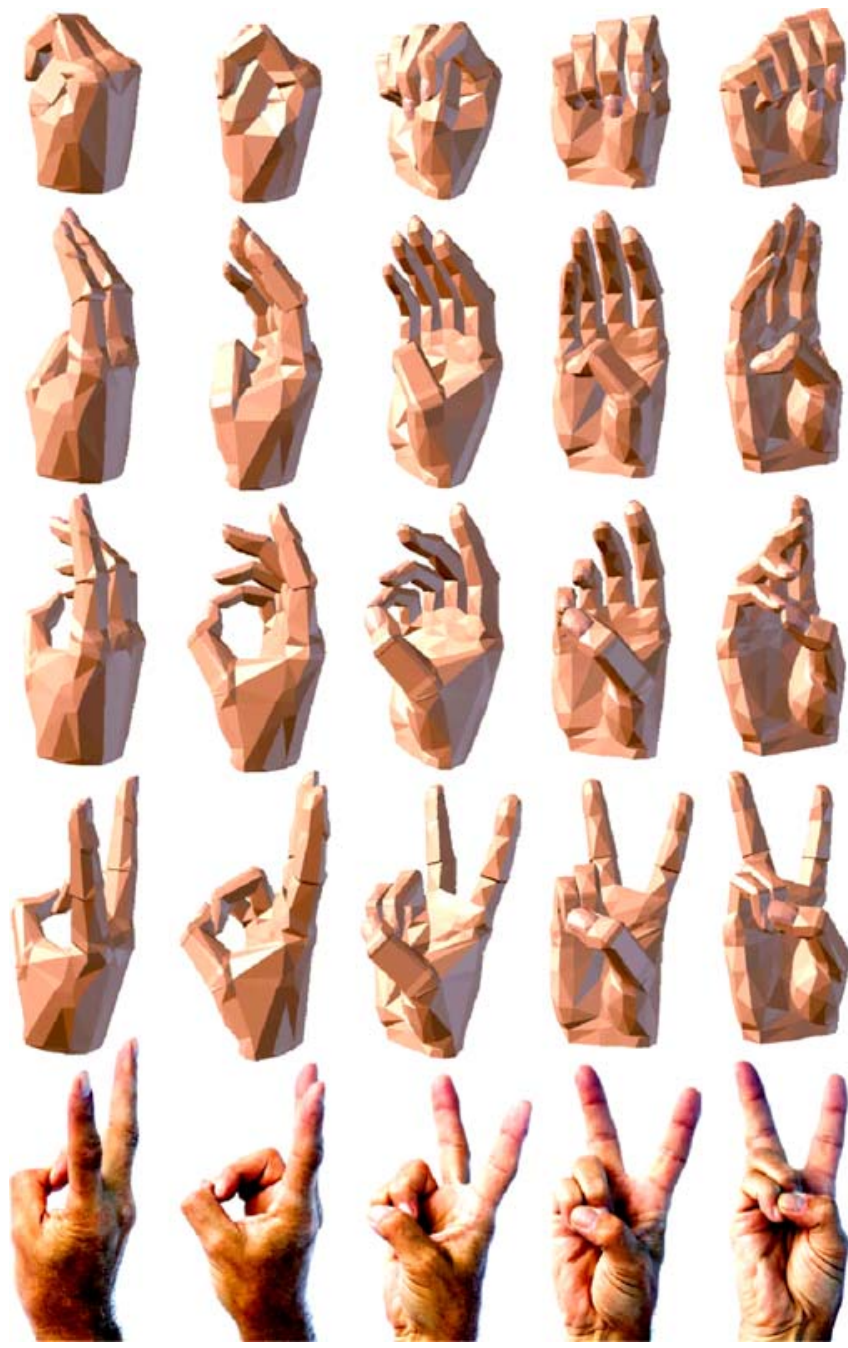

Fig. 17. 
for giving their time and insight to improve the quality of this publication.

\section{Recommendations}

- Our general recommendation to natural hand modelers is to respect the hands biological origin.

- The taxonomy can serve capturing, evaluation, adaptation and calibration of other hand models with natural motion.

- The NJA probably can model other joints in the human anatomy as well as joints in other animal life form.

- Reducing action parameters with constraints can be of special interest to animators.

- The NHHM itself can, with its low polygon count, NJA and constraints be applied to computer games, virtual manikins, sign language, hand-spelling etc.

- To capturers we have several recommendations. Capture postures from the taxonomy, if necessary more, to establish ovoid shift and locking position in individual hands.

Develop a mathematical model of the mechanical axis finder described by Hollister et al. [13].

Combine the above-mentioned with a digital photo or video system as probably then capturing and calibrating skin and biomechanics can be realized in one (relatively short) session.

For real time applications capturing motion may ask for another solution: follow the skin in direct physical contact: with data-gloves made of elastic fabrics that cling to the skin, as applied in bicycling/athletics garments (and have woven-in motion-sensors).
- Natural dynamic adaptation is perhaps the most challenging aspect of modeling natural biomechanics.

In computer visualizations natural dynamic adaptation for grasping virtual objects can be modeled with our NJA, possibly combined with the method proposed by Huang et al. [14] and with "intentional" input at the wrist, interfaced with a 3D mouse as proposed by Gribnau and Hennessy [12].

Mechanical versions of NJA and NHHM can apply dynamic adaptation to prostheses in analogy to the robothand of Bekey et al. in [22, pp. 420-421] that adapts whole fingers with amazing simple mechanics. Both implementations can enhance naturalness of prostheses and robothands by far.

\section{Future plans}

We are working on improving the accessibility of the used software to facilitate applications. And we are preparing other publications, one in which we bring forward our NHHM to the games community and another that expounds our observations on human hand modeling to the medical profession.

\section{Appendix}

The animated NHHM can be seen after loading free software from the internet and visit our website.

http://www.apple.com/quicktime/download/win.html or http://www.divx/windows/, and http://studiolab.io.tudelft.nl/vrhand/.

\section{References}

1. Albrecht, I., Haber, J., Seidel, H.-P.: Construction and animation of anatomical based human hand models. In: Proceedings of the 2003 SIGGRAPH Symposium on Computer Animation (San Diego), pp. 98-109. Eurographics Association, Aire-la-Ville, Switzerland (2003)

2. An, K.N., Chao, E.Y., Cooney, W.P., Linscheid, R.L.: Normative model of the human hand for biomechanical analysis. J. Biomechanics 12(10), 775-788 (1979)

3. Andrea, P.: Nieuw Nederlands Gebarenboekje. Elsevier Manteau, Amsterdam (1982)

4. Bando, Y., Kuratate, T., Nishita, T.: A simple method for modeling wrinkles on human skin. In: Proceedings of the 10th Pacific Conference on Computer Graphics and Applications (Beijing, China), pp. 166-175. IEEE Computer Society, Washington, DC (2002)
5. Buchholz, B., Armstrong, T.J., Goldstein, S.A.: Anthropometric data for describing the kinematics of the human hand. J. Ergonomics 35(3), 261-273 (1992)

6. Casolo, F.: In: Advances in the Biomechanics of the Hand and Wrist, Schuind, F. et al. (eds.), p. 200. NATO ASI series, Plenum Press, New York (1994)

7. Cooney, W.P. III, Lucca, M.J., Chao, E.Y.S., Linscheid, R.L.: The kinesiology of the thumb trapeziometacarpal joint. J. Bone Joint Surg. 63-A(9), 1371-1381 (1981)

8. Edwards, B.: Drawing on the right side of the brain. Tarcher, Los Angeles (1979)

9. ElKoura, G., Singh, K.: Handrix: Animating the human hand. In: Proceedings of the 2003 SIGGRAPH/Eurographics Symposium on Computer Animation (San Diego), pp. 110-119. Eurographics Association, Aire-la-Ville, Switzerland (2003)
10. Fleming, J.: Rendering tutorial. Computer Arts, 2, cover (2001)

11. Gourret, J.-P., Magnenat-Thalmann, N.M. Thalmann, D.: Simulation of object and human skin deformations in a grasping task. Comput. Graph. 23(3), 21-30 (1989)

12. Gribnau, M.W., Hennessy, J.M.: Comparing single- and two-handed 3D input for a 3D object assembly task. In: CHI '98 Conference Summary on Human Factors in Computing Systems (Los Angeles), pp. 233-234. ACM Press, New York (1998)

13. Hollister, A., Buford, W.L., Myers, L.M., Giurintano, D.J., Novick, A.: The axes of rotation of the thumb carpometacarpal joint. J. Orthopedic Res. 10(3), 454-460 (1992)

14. Huang, Z., Boulic, R., Magnenat-Thalmann, N.M., Thalmann, D.: A multi-sensor approach for grasping and 3D interaction. In: Earnshaw, R., Vince, J. (eds.) Computer Graphics: Developments in 
Virtual Environments, pp. 235-253. Academic Press Ltd., London, UK (1995)

15. Hummels, C.C.M., Stappers, P.J.: Meaningful gestures for human computer interaction: beyond hand postures. In: Proceedings of the 3rd International Conference on Face \& Gesture Recognition (Nara, Japan), pp. 591-596. IEEE Computer Society, Washington, DC (1998)

16. Kapandji, I.A.: Biomechanics of the thumb. In: The Hand, Tubiana, R. (ed.), vol. 1, Chap. 41, pp. 404-422. W.B. Saunders Company, Philadelphia (1981)

17. Kim, J., Cordier, F.,

Magnenat-Thalmann, N.M.: Neural network-based violinist's hand animation. In: Proceedings of the International Conference on Computer Graphics (Geneva, Switzerland), pp. 37-41. IEEE Computer Society, Washington, DC (2000)

18. Kuch, J.J., Huang, T.S.: Human computer interaction via the human hand: a hand model. In: 1994 Conference Record of the Twenty-Eight Asilomar Conference on Signals, Systems and Computers (Pacific Grove, CA), vol. 2, pp. 1252-1256. IEEE Computer Society, Washington, DC (1995)

19. Kurihara, T., Miyata, N.: Modeling deformable human hands from medical images. In: Proceedings of the 2004 ACM SIGGRAPH/Eurographics Symposium on Computer Animation (Grenoble, France), pp. 355-363. Eurographics Association, Aire-la-Ville, Switzerland (2004)

20. Lee, J., Kunii, T.L.: Model-based analysis of hand posture. IEEE Comput. Graph. Appl. 15(5), 77-86 (1995)

21. Lee, J., Yoon, S., Kim, M.: Realistic human hand deformation. Comput. Anim. Virtual Worlds 17, 479-489 (2006)
22. Mackenzie, C.L., Iberal, T.: The grasping hand. In: Stelmach, G.E., Vroon, P.A., $(\dagger)$ (eds.) Advances in Psychology, vol. 104. North-Holland, Amsterdam (1994)

23. Magnenat-Thalmann, N.M., Laperrière, R., Thalmann, D.: Joint dependent local deformations for hand animation and object grasping. In: Proceedings Graphics Interface '88 (Edmonton, AB, Canada), pp. 26-33. Canadian Information Processing Society, Toronto, ON, Canada (1988)

24. McDonald, J., Toro, J., Alkoby, K., Berthiaume, A., Carter, R., Chomwong, P., Christopher, J., Davidson, M.J., Furst, J., Konie, B., Lancaster, G., Roychoudhuri, L., Sedgwick, E., Tomuro, N., Wolfe, R.: An improved articulated model of the human hand. Visual Comput. 17(3), 158-166 (2001)

25. Moccozet, L., Thalmann, N.M.: Dirichlet free-form deformations and their application to hand simulation. Comput. Anim. 97, 93-102 (1997)

26. Napier, J.R.: The prehensile movement of the human hand. J. Bone Joint Surg. 38-B 902-913 (1956)

27. Rhee, T., Neumann, U., Lewis, J.P.: Human hand modeling from surface anatomy. In: Proceedings of the ACM SIGGRAPH Symposium on Interactive 3D Graphics and Games (Redwood City, CA), pp. 27-34. ACM Press, New York (2006)

28. Rijpkema, H., Girard, M.: Computer animation of knowledge-based human grasping. SIGGRAPH Comput. Graph. 25(4), 339-348 (1991)

29. Rohling, R.N., Hollerbach, J.M., Jacobsen, S.C.: Optimized fingertip mapping: a general algorithm for robotic hand teleoperation. Presence 2(3), 219 (1993)

30. Runeson, S.: On the possibility of "smart" perceptual mechanisms. Scand. J. Psychology 18, 172-179 (1977)

31. Snijders, C.J., Nordin, M., Frankel, V.H.: Biomechanica van het spier- skeletstelsel. Elzevier gezondheidszorg, Maarssen, pp. 397-400 (2001)

32. Tsang, W., Singh, K., Fiume, E.: Helping hand: an anatomically accurate inverse dynamics solution for unconstrained hand motion. In: Proceedings of the 2005 ACM SIGGRAPH/Eurographics Symposium on Computer Animation (Los Angeles), pp. 319-328. ACM Press, New York (2005)

33. Wagner, C.: The pianist's hand: anthropometry and biomechanics. Ergonomics 31(1), 97-131 (1988)

34. Williams, P.L. ( $\dagger$ ) et al.: Gray's Anatomy. Churchill Livingstone, New York (1995)

35. Wu, Y., Huang, T.S.: Human hand modelling, analysis and animation in the context of HCI. In: Proceedings of the 1999 International Conference on Image Processing, vol. 3 (Kobe, Japan), pp. 6-10. IEEE Computer Society, Washington, DC (1999)

36. Yasumuro, Y., Chen, Q., Chihara, K.: 3D modelling of human hand with motion constraints. In: Proceedings of the International Conference on Recent Advances in 3-D Digital Imaging and Modeling (Ottawa, Canada), pp. 275-282. IEEE Computer Society, Washington, DC (1997)

37. Yi, B., Harris, F.C., Wang, L., Yan, Y.: Real-time natural hand gestures. Comput. Sci. Engin. 7(3), 92-96 (2005) 

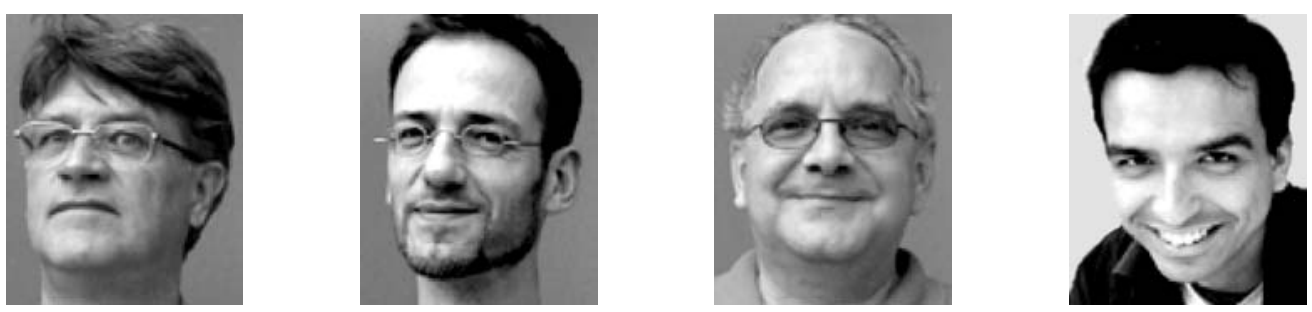

ONNO VAN NIEROP (1943) studied at the Academy for Industrial Design Eindhoven after a technical education in building construction and hydraulic engineering. From 1973 he teaches at Delft University of Technology, Department of Industrial Design. From 1985 he assisted in several research projects: "Depth through movement" and "Gestural design tools: prototypes, experiments and scenarios" the $\mathrm{PhD}$ of Caroline Hummels, which provided the basis for this project. He is a member of the IDStudioLab and has a broad research and design interest that ranges from colour and physical modeling to the "Evolution of the Bicycle".

AADJAN VAN DER HELM (1963) received a bachelor's degree in computer science from the Haagse Hogeschool in The Hague for a thesis on modeling and visualization of IFS fractals. He worked at the Computer Graphics group of Delft
University of Technology in research and education. He was involved in numerous art projects, exhibited in Europe and Australia. From 2000 he works at the Faculty of Industrial Design of the Delft University of Technology, as a scientific software engineer. He is member of the multidisciplinary group, ID-StudioLab, that investigates and designs product experience. His research interests include mathematical modeling and visualisation of natural phenomena, dataflow programming, virtual reality.

KeEs Overbeeke (1952) studied psychology at the Leuven Catholic University (Belgium). He specialized in perception and mathematical psychology. In 1988 he did his PhD at the Delft University of Technology. The title of the thesis was "Depth through movement". In this thesis a new system is described and tested to get 3D impression on a flat screen. At the moment he works at the Faculty of Industrial Design of the Eindhoven University of Technology as associate professor. His research and teaching interests include design \& emotion, expressivity and experience of products, and the resulting new philosophy of science and methodology, 3D perception, eye-hand coordination.

TOM DJAJADININGRAT (1968) studied industrial design engineering at Brunel University and the Royal College of Art. He became interested in virtual reality during his $\mathrm{PhD}$ study at Delft University. After some years at the Faculty of Industrial Design of the Eindhoven University of Technology, trying to combine the real and virtual in product design with a special interest in tangible interaction and augmented reality, he is now working at the Philips Design department in Eindhoven. 